\title{
The association between statins and colorectal cancer stage in the Women's Health Initiative
}

\author{
BRIAN P. RUTLEDGE ${ }^{1}$, PINKAL DESAI ${ }^{2}$, SIMIN LIU $^{3}$, JUHUA LUO ${ }^{4}$, RAMI NASSIR $^{5}$, \\ QI LIHONG ${ }^{6}$, MONICA ARUN ${ }^{7}$, MAHMOUD ABDEL-RASOUL $^{8}$ and MICHAEL S. SIMON ${ }^{9}$ \\ ${ }^{1}$ Department of Internal Medicine, Wayne State University School of Medicine, Detroit, MI 48201; \\ ${ }^{2}$ Department of Hematology and Medical Oncology Weill Cornell Medical College, New York, NY 10065; \\ ${ }^{3}$ The Alpert School of Medicine, Center for Global Cardiometabolic Heath, and School of Public Health, \\ Brown University, Providence, RI 02093; ${ }^{4}$ School of Public Health, Indiana University, Bloomington, IN 47405; \\ Departments of ${ }^{5}$ Biochemistry and Molecular Medicine and ${ }^{6}$ Public Health Sciences, University of California, \\ Davis, CA 95817; ${ }^{7}$ Department of Internal Medicine, Division of Hematology and Onology, \\ University of Michigan Medical School, Ann Arbor, MI 48109; ${ }^{8}$ Department of Biomedical Informatics, \\ College of Medicine, Ohio State University, Columbus, OH 43201; ${ }^{9}$ Department of Oncology, \\ Karmanos Cancer Institute, Wayne State University, Detroit, MI 48201, USA
}

Received September 6, 2018; Accepted June 24, 2019

DOI: $10.3892 / \mathrm{mco} .2019 .1895$

\begin{abstract}
The anticarcinogenic effect of statins may reduce the metastatic potential of cancer cells leading to 'stage migration', with users more likely diagnosed with early rather than late stage cancer. The association between prior statin use and colorectal cancer (CRC) stage at diagnosis in the Women's Health Initiative (WHI) was investigated. The study population included 132,322 post-menopausal women, among which there were 2,628 pathologically confirmed cases of in situ (3.3\%), localized (43.6\%), regional (40.4\%) and distant (12.7\%) stage CRC, after an average of $13.9(\mathrm{SD}=4.7)$ years of follow-up. To reduce the possibility of detection bias among women more likely to be prescribed statins, women who did not report a mammogram within 5 years of study entry and who had no health insurance or medical care provider $(n=28,237)$ were excluded from the study. Stage was coded using SEER criteria into early (in situ and local) vs. late (regional and distant) stage disease. Hazards ratios (HR) and $95 \%$ confidence intervals (CIs) evaluating the association between statin use and diagnosis of late-stage CRC both at baseline and in a time-dependent manner were computed from multivariable-adjusted Cox proportional hazards analyses. In the multivariable time-dependent analysis, there was a lower hazard of late stage CRC among users of lipophilic statins
\end{abstract}

Correspondence to: Dr Brian P. Rutledge, Wayne State University School of Medicine, Harper University Hospital, 3990 John R St., Room no. 6938, Detroit, MI 48201, USA

E-mail: brianrutledge87@gmail.com; brian.rutledge@wayne.edu

Key words: hydroxymethylglutaryl-CoA reductase inhibitors, colorectal neoplasm, neoplasm staging, hydrophobic, hydrophilic compared with non-users $(\mathrm{HR}=0.80,95 \%$ CI 0.66-0.98, $\mathrm{P}=0.029$ ) and a marginally lower hazard of late stage CRC among users of lipophilic vs. hydrophilic statins $(\mathrm{HR}=0.70$, 95\% CI 0.49-1.01, $\mathrm{P}=0.058$ ). The use of lipophilic statins was associated with a reduction in the proportion of CRC cases that were late stage at the time of diagnosis.

\section{Introduction}

Statins are widely prescribed in the United States with up to $25 \%$ of the population over age 45 estimated to use the medications from 2005 to 2008 (1). This is largely attributed to the demonstrated impact of statins on cardiovascular events and mortality in several randomized controlled trials (2-4). Over 62 million individuals are estimated to be statin-eligible based on guidelines from the ACC/AHA for statin use (5).

Statins are well known as inhibitors of 3-hydroxy-3-methyl glutaryl coenzyme A (HMG-CoA) reductase, the enzyme required for the conversion of HMG-CoA to mevalonic acid (6) and a number of cancers, including colorectal cancer (CRC), have molecular pathways which are potentially affected by the inhibition of mevalonic acid synthesis as well as through alternative pathways $(7,8)$. Statins have also been shown to arrest cell cycle progression, to alter the adhesion and migration of tumor cells, and to induce tumor cell apoptosis potentially leading to a reduced risk of metastasis (9-11).

A number of epidemiologic studies, including one utilizing the Women's Health Initiative (WHI) database, have assessed the relationship between statins and CRC risk and have shown mixed results with some studies reporting a protective effect (12-14) and others no association $(15,16)$. In particular, a previous study utilizing the WHI database has addressed the specific question of whether statins have an effect on the risk of CRC diagnosis and the results did show a benefit for the lipophilic sub-type of statins (12). Other studies including 
two recent meta-analyses have revealed a generally weak influence of statin use on overall survival in patients with CRC with an inconsistent reduction in CRC specific mortality (17-19). There is only one prior study to date that has looked at the specific hypothesis of this study which is the relationship between statins and CRC stage at diagnosis. That study reported that 3 or more years of pre-diagnosis statin use was associated with lower AJCC tumor stage and lower prevalence of metastases compared to non-users (20).

In the current analysis, we evaluated whether prior statin use had an impact on CRC stage at the time of cancer diagnosis using data from the Women's Health Initiative (WHI) cohort. Our specific hypothesis for this study was to determine whether prior statin use had an impact on stage of CRC at diagnosis. The literature suggests that through the inhibition of cell migration and angiogenesis, along with reported pro-apoptotic effects, statins are hypothesized to have anti-invasive, anti-proliferative, and ultimately anti-metastatic effects $(9-11,18,21-26)$. We hypothesized that the anticancer effects of statins would have a potential impact on CRC stage at diagnosis.

\section{Materials and methods}

Studypopulation. The study population included 161,806 women enrolled in either the WHI clinical trials (CT) $(n=68,132)$ or observational study (OS) $(n=93,676)$ and included women with newly diagnosed incident invasive and in situ CRC through the end of the first WHI Extension Study. The CT consisted of randomized trials of hormone therapy, dietary modification, and/or calcium and vitamin D supplementation $(27,28)$. More information related to the WHI trials including the study's design, procedures, and components can be found at the WHI website (29). In order to reduce the possibility of detection bias among women more likely to be prescribed statins, we excluded from the analysis women who did not report a mammogram within 5 years of study entry $(16,686)$, women with no health insurance at baseline $(5,732)$, and women with no reported medical care provider $(5,818)$. We also excluded women who had a prior history of CRC (813) and women with missing information on baseline statin use (2) resulting in a total of 29,051 women who were excluded from the analysis. We did not exclude participants based on whether or not they had a colonoscopy within the past 10 years as this was thought to limit the size of the study population number in a time period where alternative methods of CRC screening such as sigmoidoscopy were part of the standard of care. In total, there were 132,757 women included in the analysis who were followed for an average of 13.9 (SD 4.72) years.

Statin exposure. Statin use was defined as use of any HMG-CoA reductase inhibitor. Statins are classified as either lipophilic or hydrophilic. This classification is based on their solubility in octanol (lipophilicity) or water (hydrophilicity). Corresponding to their solubility properties, lipophilic statins penetrate the plasma membrane while hydrophilic statins do not (30).

Statin exposure was defined as statin use for any duration of time before the diagnosis of CRC. We analyzed baseline statin exposure from CT and OS participants as well as follow-up information on statin use determined at year 3 in the OS and years 1, 3, 6 and 9 in the CT, and statin use at the start of the $2^{\text {nd }}$ extension study for both $(27,28)$. At baseline and each follow-up period, participants were asked to bring all of their current prescription medications to the clinic visit (or first interview at baseline). At those times, study personnel entered each medication name directly from the medication containers into the WHI database, which assigned drug codes using Medispan software (First DataBank, Inc.). At the time of the visit, women also reported duration of use for each current medication. Information related to statin use at subsequent visits continued to be ascertained throughout the study and was used to develop a time dependent measure of statin exposure in this study.

Covariates. Variables within the study population that can affect risk of CRC may have an effect on the stage migration and were assessed as potential confounding variables and are listed in Table I. Information on these variables was collected on the baseline WHI study questionnaires and included participant socio-demographics, medical history and other information on established risk factors for CRC $(17,27,28)$.

Outcomes. Cancer diagnoses were updated by telephone questionnaires and/or by mail semi-annually in the CT and annually in the OS. Centrally trained physician adjudicators were utilized to verify participant or next of kin reports of CRC through careful review of pathology reports and supporting medical records in conjunction with the Surveillance Epidemiology and End Results (SEER) coding system. Information on the frequency of screening tests, including fecal occult blood tests, rectal examinations, and sigmoidoscopy or colonoscopy was collected at baseline and updated every 6 months in the CT and every 12 months in the OS. Corresponding to the goals of this study, stage was stratified as either early-stage disease (in situ and local) versus late-stage (regional and distant) disease. There was a total of 13.9 years of follow-up (SD 4.7 years) from the start through the end of the first WHI Extension Study with $(n=2,628)$ centrally adjudicated and SEER-coded CRC cases [89 in situ (3.25\%), 1,145 localized stage (42.8\%), 1,062 regional stage (39.6\%) and 334 distant stage (12.4\%).

Statistical analysis. Univariate and multivariable Cox models were fit to assess the relationship between each of overall statin use at baseline, type of statin use at baseline, and duration of statin use at baseline respectively with time to late stage CRC diagnosis as the outcome. Women who died during follow-up in the study or presented with early stage CRC were treated as censored in all of the main analyses' models. The baseline hazard for the univariate and multivariable models was stratified both by WHI trial membership and age stratum. Multivariable models were created using the following clinically relevant covariates determined a priori as covariates: race, education, smoking, alcohol, body mass index (BMI), mammogram in the past 2 years, aspirin use, and history of colonoscopy or sigmoidoscopy.

All covariates in the multivariable models were categorized as seen in Table I. Statin use at baseline was categorized by use vs. non-use, type (lipophilic vs. hydrophilic), and duration of use ( $<1$ year, 1-3 years, and $3+$ years) for each of the respective 
Table I. Selected demographics and clinical characteristics by baseline statin use in the Women's Health Initiative.

\begin{tabular}{l} 
Variable \\
\hline Age group at enrollment (years) \\
$50-54$ \\
$55-59$ \\
$60-69$ \\
$70-79+$
\end{tabular}

$$
\begin{gathered}
\text { No baseline statin use } \\
\mathrm{n}=121,889(92 \%)
\end{gathered}
$$

Baseline statin use $\mathrm{n}=10,868(8 \%)$
Ethnicity

Native American/Alaskan native

Asian or Pacific Islander

Black or African American

Hispanic or Latino

White (not of Hispanic origin)

Other

Education

None to some HS

HS diploma/GED

$16,414(13 \%)$

$24,166(20 \%)$

$54,527(45 \%)$

$26,782(22 \%)$

$$
\begin{array}{rr}
464 & (0 \%) \\
3,135 & (3 \%) \\
9,801 & (8 \%) \\
3,578 & (3 \%) \\
103,292 & (85 \%) \\
1,320 & (1 \%)
\end{array}
$$

$4,877 \quad(4 \%)$

$19,925(16 \%)$

45,397 (37\%)

some college or associate degree

College graduate or more

BMI $\left(\mathrm{kg} / \mathrm{m}^{2}\right)$

$<25$

25-29

$>30$

Smoking

Never smoked

Past smoker

Current smoker

Alcohol use

Non-drinker or past drinker

$<1$ drink/month to $<7$ drinks per week

$7+$ drinks per week

Overall physical activity

None

$>0$ to 3.75 MET-hours/week

3.75-8.75 MET-hours/week

8.75-17.5 MET-hours/week

$>17.5$ MET-hours/week

Aspirin use

No

Yes

Mammogram within 2 years

No

Yes

Colon screening

Yes, $<5$ years ago

Yes, $>5$ years ago

Never
$50,996(42 \%)$

$44,560(37 \%)$

$41,804(34 \%)$

34,464 (28\%)

$61,771(51 \%)$

$51,463(42 \%)$

$7,189 \quad(6 \%)$

$33,733(28 \%)$

$72,460(59 \%)$

$14,892(12 \%)$

$17,191(14 \%)$

$16,344(13 \%)$

$23,759(19 \%)$

$27,095(22 \%)$

$31,793(26 \%)$

$98,423(81 \%)$

23,466 (19\%)

$10,709(9 \%)$

$111,180(91 \%)$

40,693 (35\%)

$22,221(19.2 \%)$

$52,606(46 \%)$
$575 \quad(5 \%)$

$1,346(12 \%)$

$5,694(52 \%)$

$3,253(30 \%)$

$34 \quad(0 \%)$

$434 \quad(4 \%)$

$904 \quad(8 \%)$

$289(3 \%)$

$9,057(83 \%)$

$121 \quad(1 \%)$

$636 \quad(6 \%)$

$2,268(21 \%)$

4,197 (39\%)

$3,708(34 \%)$

$2,723(25 \%)$

$4,318(40 \%)$

3,737 (34\%)

$5,263(48 \%)$

$4,852(45 \%)$

$605(6 \%)$

$3,628(33 \%)$

6,137 (56\%)

1,037 (10\%)

1,501 (14\%)

$1,614(15 \%)$

2,318 (21\%)

$2,515(23 \%)$

$2,658(24 \%)$

$7,122(66 \%)$

$3,746(34 \%)$

$744(7 \%)$

$10,124(93 \%)$

4,154 (40\%)

$2,159(20 \%)$

4,228 (40\%) univariate and multivariable models assessing the relationships between these variables and time to late stage CRC diagnosis.
To examine the impact of change in statin use over time, univariate and multivariable Cox models were created with 
Table II. Characteristics of statin use and CRC outcomes

\begin{tabular}{lrl}
\hline Characteristic & Number $(\%)$ \\
\hline Baseline statin use: & & \\
No & 121,889 & $(92 \%)$ \\
Yes & 10,868 & $(8 \%)$ \\
Baseline statin duration ${ }^{\mathrm{a}}:$ & & \\
No baseline statin use & 121,889 & $(92 \%)$ \\
$<1$ year & 3,541 & $(3 \%)$ \\
1-3 years & 3,711 & $(3 \%)$ \\
$3+$ years & 3,616 & $(3 \%)$ \\
Baseline statin name: & & \\
No baseline statin use & 121,889 & $(92 \%)$ \\
Atorvastatin calcium & 837 & $(1 \%)$ \\
Fluvastatin sodium & 1,330 & $(1 \%)$ \\
Lovastatin & 2,955 & $(2 \%)$ \\
Pravastatin sodium & 2,456 & $(2 \%)$ \\
Simvastatin & 3,290 & $(2 \%)$ \\
Baseline statin type: & & \\
None & $121,889(92 \%)$ \\
Lipophilic & 8,412 & $(6 \%)$ \\
Hydrophilic & 2,456 & $(2 \%)$ \\
\hline
\end{tabular}

${ }^{\mathrm{a}}>100 \%$ due to rounding. CRC, colorectal cancer.

statin use (by type and by use vs. non-use) as time dependent exposures. Time varying statin use models (overall and by statin type) were based on updates on statin intake obtained at follow-up clinic visits. The time varying models were also categorized by age stratum at randomization and WHI trial membership, and used the same covariates as the baseline models. All analyses were performed using SAS/STAT software version 9.4 (SAS Institute Inc). P-values less than 0.05 were considered to be statistically significant. We also fit competing risk models treating both diagnosis of early stage $\mathrm{CRC}$ and death as competing risks respectively as sensitivity analyses for all of the main analyses' models described above.

\section{Results}

The study consisted of 10,868 women who reported statin use at baseline and 121,889 who reported no statin use at baseline. The mean age of the postmenopausal population was $\sim 63$ years old and $84.6 \%$ of the study participants were white. Table I describes the demographic and clinical characteristics of the study cohort. Statin users were more likely than non-users to be older, diabetic, overweight or obese; however, family history of CRC, tobacco use, and alcohol use were relatively similar between the two groups. Statin users were also slightly more likely to have had a screening colonoscopy and to be taking aspirin or an NSAID. Table II describes the distribution of statin use at baseline by duration, type of statin used, and other statin characteristics. Additionally, Table II lists the number of CRC cases both by severity and also in association with statin use at baseline. It is important to note however that the data in Table II is included to demonstrate the baseline data on statin use by stage of CRC, and does not include data pertinent to the time dependent analysis.

Table III shows the relationship between late-stage CRC at diagnosis and statin use. In the multivariable model, there was no significant association between statin use at baseline and late-stage CRC ( $\mathrm{HR}=1.08$; 95\% CI, (0.86-1.36, $\mathrm{P}=0.498)$. Table IV shows the relationship between late-stage CRC and statin use in a time-dependent model. In the multivariable time-dependent analyses, overall statin use (regardless of type) was not associated with a significant reduction in late-stage CRC (HR=0.87, 95\% CI, 0.73-1.03, P=0.109). However, when statin type was assessed, there was found to be a significant relationship between lipophilic statin use and late stage CRC $(\mathrm{HR}=0.80,95 \% \mathrm{CI}, 0.66-0.98, \mathrm{P}=0.029)$ compared to no statin use over time. When comparing lipophilic vs. hydrophilic statin use there was a marginally decreased risk of late stage CRC for users of lipophilic statins, however these differences were not statistically significant $(\mathrm{HR}=0.70,95 \% \mathrm{CI}, 0.49-1.01$, $\mathrm{P}=0.058)$.

Several sensitivity analyses were performed. First, we checked for possible selection bias by repeating our baseline statin use multivariable models for late-stage CRC without any exclusion for healthcare access; indicator variables for the original inclusion criteria (mammogram in the past 5 years, current health insurance, current healthcare provider and no personal history of CRC) were added as additional covariates in the multivariable models. The hazard ratio for statin use at baseline (yes vs. no) from the multivariable model using the extended cohort was 1.02 compared to the original hazard ratio show in Table III of 1.08. We further assessed whether treating diagnosis of early stage $\mathrm{CRC}$ or death as competing risks would have an effect on the cox model estimates, and found the hazard ratios to be similar to the initial models.

\section{Discussion}

The primary goal of this study was to determine whether prior statin use is associated with earlier stage CRC at the time of cancer diagnosis. While our analysis of statin use at baseline alone showed no significant association, after taking into account subsequent exposure to statins captured in a time-dependent analysis, we found a significant reduction in the proportion of cancer diagnoses that were consistent with late stage disease among users of statins. Our findings appeared to depend on the type of statin used, as specifically users of lipophilic statins were found to have decreased risk for late stage disease when analyzed independently compared to non-users. The impact of hydrophilic statins which, in contrast, did not have a protective association, is likely responsible for the aggregate statin group results lack of a statistically significant association. In a similar analysis, we reported a reduction in late stage breast cancer among prior users of statins as well as a marginally lower risk of death from breast cancer among users of lipophilic statins (31). In another previous WHI analysis assessing the overall risk of CRC in statin users, a significant reduction in CRC risk for lovastatin, a lipophilic statin, was observed (12). Given our prior findings, the present study was designed to specifically focus on the hypothesis that there is a reduced likelihood of advanced stage CRC in statin users at 
Table III. CRC stage migration by baseline statin use.

\begin{tabular}{lllll}
\hline Model type & \multicolumn{1}{c}{ Comparison } & HR & 95\% CI & P-value \\
\hline Univariable: baseline statin (Y/N) & Baseline statin (yes vs. no) & 1.06 & $(0.85,1.32)$ & 0.628 \\
Univariable: baseline statin duration & Baseline statin: <1 year vs. none & 0.88 & $(0.58,1.33)$ & 0.548 \\
& Baseline statin: 1-3 years vs. none & 1.32 & $(0.95,1.83)$ & 0.100 \\
& Baseline statin: 3+ years vs. none & 0.96 & $(0.65,1.41)$ & 0.830 \\
& Baseline statin: <1 year vs. 1-3 years & 0.67 & $(0.40,1.12)$ & 0.129 \\
& Baseline statin: <1 year vs. 3+ years & 0.92 & $(0.53,1.60)$ & 0.764 \\
& Baseline statin: 1-3 years vs. 3+ years & 1.38 & $(0.84,2.26)$ & 0.208 \\
& Baseline statin: lipophilic vs. none & 0.96 & $(0.74,1.24)$ & 0.733 \\
Univariable: baseline statin type & Baseline statin: hydrophilic vs. none & 1.41 & $(0.95,2.08)$ & 0.088 \\
& Baseline statin: lipophilic vs. hydrophilic & 0.68 & $(0.43,1.08)$ & 0.100 \\
& Baseline statin (yes vs. no) & 1.08 & $(0.86,1.36)$ & 0.498 \\
Multivariable & Baseline statin: <1 year vs. none & 0.86 & $(0.56,1.33)$ & 0.490 \\
Multivariable: baseline statin duration & Baseline statin: 1-3 years vs. none & 1.43 & $(1.02,1.99)$ & 0.036 \\
& Baseline statin: 3+ years vs. none & 0.95 & $(0.64,1.42)$ & 0.814 \\
& Baseline statin: <1 year vs. 1-3 years & 0.60 & $(0.35,1.03)$ & 0.063 \\
& Baseline statin: <1 year vs. 3+ years & 0.90 & $(0.50,1.61)$ & 0.723 \\
& Baseline statin: 1 -3 years vs. 3+ years & 1.50 & $(0.90,2.49)$ & 0.120 \\
& Baseline statin: lipophilic vs. none & 1.01 & $(0.78,1.32)$ & 0.943 \\
Multivariablea ${ }^{a}$ baseline statin type & Baseline statin: hydrophilic vs. none & 1.33 & $(0.88,2.02)$ & 0.177 \\
& Baseline statin: lipophilic vs. hydrophilic & 0.76 & $(0.47,1.23)$ & 0.259 \\
\hline
\end{tabular}

${ }^{a}$ Adjusted for race, education, smoking, BMI, mammogram in the past 2 years, aspirin use, and history or colonoscopy. History of colonoscopy is a categorical variable that has the following categories (no, yes, within the past 5 years; yes, more than 5 years ago). CRC, colorectal cancer; HR, hazard ratio; CI, confidence interval.

Table IV. Association of statin use over time and late stage CRC stratified by type of statin.

\begin{tabular}{llccr}
\hline Model type & \multicolumn{1}{c}{ Comparison } & HR & 95\% CI & P-value \\
\hline Univariable: Statin use over time (Y/N) & Statin use over time (yes vs. no) & 0.85 & $(0.72,1.01)$ & 0.064 \\
Multivariable & Statin use over time (yes vs. no) & 0.87 & $(0.73,1.03)$ & 0.109 \\
Univariable: Statin use over time: & Statin use over time: lipophilic vs. none & 0.79 & $(0.66,0.96)$ & 0.016 \\
by type & Statin use over time: hydrophilic vs. none & 1.18 & $(0.86,1.61)$ & 0.303 \\
& Statin use over time: lipophilic vs. hydrophilic & 0.67 & $(0.48,0.96)$ & 0.027 \\
Multivariablea: Statin use & Statin use over time: lipophilic vs. none & 0.80 & $(0.66,0.98)$ & 0.029 \\
over time: by type & Statin use over time: hydrophilic vs. none & 1.14 & $(0.83,1.59)$ & 0.419 \\
& Statin use over time: lipophilic vs. hydrophilic & 0.70 & $(0.49,1.01)$ & 0.058 \\
\hline
\end{tabular}

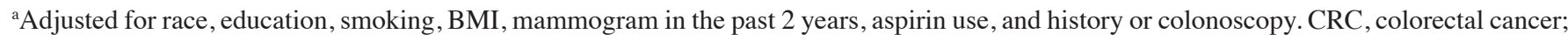
HR, hazard ratio; CI, confidence interval.

the time of diagnosis due to the anticarcinogenic molecular pathways associated with statin use. The results from our prior work adds evidence to the hypothesis of a preferential effect of lipophilic statins compared to hydrophilic statins possibly related to the increased permeability of lipophilic statins across the cell membrane.

In order to have a beneficial impact on CRC outcomes, it is desirable to identify strategies to either prevent or down-stage disease at diagnosis $(32,33)$. Through the inhibition of cell migration and angiogenesis, along with reported pro-apoptotic effects, statins are hypothesized to have anti-invasive, anti-proliferative, and ultimately anti-metastatic effects $(9-11,18,21-26)$. There has only been one other population-based study that has looked at the relationship between statin use and CRC stage at diagnosis however others have also assessed the impact of statins on response to adjuvant or neoadjuvant chemotherapy (18,19,34-36). Among 1,309 male veterans with CRC, use of 3 or more years of pre-diagnosis 
statins was associated with a lower mean AJCC tumor stage (2.2 for users vs. 2.6 for non-users; $\mathrm{P}<0.01$ ) and lower prevalence of metastases [OR $=0.7(0.4-0.9,95 \% \mathrm{CI})$; P<0.01] (20). Also, in an analysis of 407 individuals with primary rectal cancer, statin use was significantly associated with improved response to chemotherapy as measured by the AJCC tumor regression grading system which itself was associated with improved oncologic outcomes (35) results of which were collaborated by others (34). The results of our study are consistent with others in the literature suggesting the possibility that, through their previously described inhibition of the mevalonic acid pathway, statins may have a mitigatory effect on the severity or stage of cancer found at the time of diagnosis.

The strengths of our analysis include the WHI protocol for comprehensive assessment and central review of cancer diagnoses with accompanying information related to demographics and cancer risk factors. Limitations include an inability to utilize the TNM classification system for staging due to missing data on the number of involved lymph nodes at the time of diagnosis. Notably, in addition there was a much lower prevalence of statin use at the study's inception as compared with predicted current use (1) and we were unable to confirm medication start dates or compliance. There is also the potential for other biases given that statin users may be more likely to have had better medical care and to have exposure to CRC screening which may account for earlier stage at diagnosis among statin users. We attempted to control for bias related to access to care by only including women in the analysis who had a mammogram in the past 5 years and who had health insurance and a regular medical provider and we also adjusted our results for past reported colon cancer screening. However, the dataset was not comprehensive in relation to the frequency of or indication for colonoscopic evaluation. Without additional information on screening colonoscopy, we were unable to construct a meaningful time-dependent control for screening colonoscopy. The difficulties with both construction of a comprehensive medication use history as well as construction of a detailed history of screening colonoscopy reflect the retrospective nature of our analysis within the setting of an observational study. In light of these limitations, in our view the results of the study should be viewed primarily as exploratory and hypothesis provoking. Further investigation by clinical trial would aid in a more definitive conclusion.

In summary, our results suggest that lipophilic statins use may be associated with an anticarcinogenic effect corresponding with a lower likelihood of developing late stage CRC which may have future implications for CRC prevention and management.

\section{Acknowledgements}

We acknowledge the dedicated efforts of investigators and staff at the Women's Health Initiative (WHI) clinical centers, the WHI Clinical Coordinating Center, and the National Heart, Lung and Blood program office (listing available at http://www.whi.org). We also recognize the WHI participants for their extraordinary commitment to the WHI program. For a list of all the investigators who have contributed to WHI science, please visit: http://www.whiscience.org/publications/WHI_investigators_longlist.pdf. This abstract was presented at the American Society of Clinical Oncology in
Chicago, IL, USA on June 5th, 2017, and was published as Abstract no. 1540.

\section{Funding}

The WHI program is funded by the National Heart, Lung, and Blood Institute, National Institutes of Health, U.S. Department of Health and Human Services through contracts N01WH22110, 24152, 32100-2, 32105-6, 32108-9, 32111-13, 32115, 32118-32119, 32122, 42107-26, 42129-32, and 44221", and the Cancer Center Support Grant NIH:NCI P30CA022453.

\section{Availability of data and materials}

The data that support the findings of this study are available from the National Heart, Lung and Blood Institute (https://biolincc-nhlbi-nih-gov/studies/whios/), but restrictions apply to the availability of these data. Data are however available from the WHI upon reasonable request.

\section{Authors' contributions}

BPR and MSS were responsible for the conception, design, and interpretation of data analysis and drafting of the manuscript. MAR was responsible for the data analysis. PD, SL, JL, RN, QL and MA made substantial contributions to the analysis and interpretation of the data along with being responsible for the revisions and critical review of the drafts. All authors have approved the final version of this manuscript.

\section{Ethics approval and consent to participate}

The Women's Health Initiative was overseen by ethics committee at all 40 clinical centers, and an independent data and safety monitoring board for the clinical trials. Each institution obtained human subjects committee approval. Each participant provided written informed consent.

\section{Patient consent for publication}

Not applicable.

\section{Competing interests}

The authors declare that they have no competing interests.

\section{References}

1. Gu Q, Paulose-Ram R, Burt VL and Kit BK: Prescription cholesterol-lowering medication use in adults aged 40 and over: United States, 2003-2012. National Center for Health Statistics. 2014. NCHS Data Brief 177: 1-8, 2014

2. Sacks FM, Pfeffer MA, Moye LA, Rouleau JL, Rutherford JD, Cole TG, Brown L, Warnica JW, Arnold JM, Wun CC, et al: The effect of pravastatin on coronary events after myocardial infarction in patients with average cholesterol levels. Cholesterol and Recurrent Events Trial investigators. N Engl J Med 335: 1001-1009, 1996.

3. Long-Term Intervention with Pravastatin in Ischaemic Disease (LIPID) Study Group: Prevention of cardiovascular events and death with pravastatin in patients with coronary heart disease and a broad range of initial cholesterol levels. N Engl J Med 339: 1349-1357, 1998 
4. Serruys PW, de Feyter P, Macaya C, Kokott N, Puel J, Vrolix M, Branzi A, Bertolami MC, Jackson G, Strauss B, et al; Lescol Intervention Prevention Study (LIPS) Investigators: Fluvastatin for prevention of cardiac events following successful first percutaneous coronary intervention: A randomized controlled trial JAMA 287: 3215-3222, 2002

5. Wong ND, Young D, Zhao Y, Nguyen H, Caballes J, Khan I and Sanchez RJ: Prevalence of the American College of Cardiology/American Heart Association statin eligibility groups, statin use, and low-density lipoprotein cholesterol control in US adults using the National Health and Nutrition Examination Survey 2011-2012. J Clin Lipidol 10: 1109-1118, 2016.

6. Chan KK, Oza AM and Siu LL: The statins as anticancer agents. Clin Cancer Res 9: 10-19, 2003.

7. Ishikawa S, Hayashi H, Kinoshita K, Abe M, Kuroki H, Tokunaga R, Tomiyasu S, Tanaka H, Sugita H, Arita T, et al: Statins inhibit tumor progression via an enhancer of zeste homolog 2-mediated epigenetic alteration in colorectal cancer. Int J Cancer 135: 2528-2536, 2014

8. Bardou M, Barkun A and Martel M: Effect of statin therapy on colorectal cancer. Gut 59: 1572-1585, 2010.

9. Wong WW, Dimitroulakos J, Minden MD and Penn LZ: HMG-CoA reductase inhibitors and the malignant cell: The statin family of drugs as triggers of tumor-specific apoptosis Leukemia 16: 508-519, 2002.

10. Keyomarsi K, Sandoval L, Band V and Pardee AB Synchronization of tumor and normal cells from G1 to multiple cell cycles by lovastatin. Cancer Res 51: 3602-3609, 1991.

11. Mehta N, Hordines J, Sykes D, Doerr RJ and Cohen SA: Low density lipoproteins and Lovastatin modulate the organ-specific transendothelial migration of primary and metastatic human colon adenocarcinoma cell lines in vitro. Clin Exp Metastasis 16 587-594, 1998.

12. Simon MS, Rosenberg CA, Rodabough RJ, Greenland P, Ockene I, Roy HK, Lane DS, Cauley JA and Khandekar J: Prospective analysis of association between use of statins or other lipid-lowering agents and colorectal cancer risk. Ann Epidemiol 22: 17-27,2012.

13. Poynter JN, Gruber SB, Higgins PD, Almog R, Bonner JD, Rennert HS, Low M, Greenson JK and Rennert G: Statins and the risk of colorectal cancer. N Engl J Med 352: 2184-2192, 2005.

14. Lytras T, Nikolopoulos G and Bonovas S: Statins and the risk of colorectal cancer: an updated systematic review and meta-analysis of 40 studies. World J Gastroenterol 20: 1858-1870, 2014.

15. Bonovas S, Filioussi K, Tsavaris N and Sitaras NM: Statins and cancer risk: A literature-based meta-analysis and meta-regression analysis of 35 randomized controlled trials. J Clin Oncol 24 4808-4817, 2006.

16. Bonovas S, Filioussi K, Flordellis CS and Sitaras NM: Statins and the risk of colorectal cancer: A meta-analysis of 18 studies involving more than 1.5 million patients. J Clin Oncol 25: 3462-3468, 2007.

17. Cai H, Zhang G, Wang Z, Luo Z and Zhou X: Relationship between the use of statins and patient survival in colorectal cancer: A systematic review and meta-analysis. PLoS One 10 e0126944, 2015.

18. Gray RT, Coleman HG, Hughes C, Murray LJ and Cardwell CR: Statin use and survival in colorectal cancer: Results from a population-based cohort study and an updated systematic review and meta-analysis. Cancer Epidemiol 45: 71-81, 2016.

19. Cardwell CR, Hicks BM, Hughes C and Murray LJ: Statin use after colorectal cancer diagnosis and survival: A population-based cohort study. J Clin Oncol 32: 3177-3183, 2014.

20. Siddiqui AA, Nazario H, Mahgoub A, Patel M, Cipher D and Spechler SJ: For patients with colorectal cancer, the long-term use of statins is associated with better clinical outcomes. Dig Dis Sci 54: 1307-1311, 2009.

21. Seasholtz TM, Majumdar M and Brown JH: Rho as a mediator of $G$ protein-coupled receptor signaling. Mol Pharmacol 55: 949-956, 1999

22. Holstein SA, Wohlford-Lenane CL and Hohl RJ: Isoprenoids influence expression of Ras and Ras-related proteins. Biochemistry 41: 13698-13704, 2002.
23. Denoyelle C, Vasse M, Körner M, Mishal Z, Ganné F, Vannier JP, Soria J and Soria C: Cerivastatin, an inhibitor of HMG-CoA reductase, inhibits the signaling pathways involved in the invasiveness and metastatic properties of highly invasive breast cancer cell lines: An in vitro study. Carcinogenesis 22: 1139-1148, 2001.

24. Sleijfer S, van der Gaast A, Planting AS, Stoter G and Verweij J: The potential of statins as part of anti-cancer treatment. Eur J Cancer 41: 516-522, 2005.

25. Nübel T, Dippold W, Kleinert H, Kaina B and Fritz G: Lovastatin inhibits Rho-regulated expression of E-selectin by TNFalpha and attenuates tumor cell adhesion. FASEB J 18: 140-142, 2004.

26. Cho SJ, Kim JS, Kim JM, Lee JY, Jung HC and Song IS: Simvastatin induces apoptosis in human colon cancer cells and in tumor xenografts, and attenuates colitis-associated colon cancer in mice. Int J Cancer 123: 951-957, 2008.

27. Anderson GL, Manson J, Wallace R, Lund B, Hall D, Davis S, Shumaker S, Wang CY, Stein E and Prentice RL: Implementation of the women's health initiative study design. Ann Epidemiol 13 (Suppl): S5-S17, 2003.

28. Langer RD, White E, Lewis CE, Kotchen JM, Hendrix SL and Trevisan M: The Women's Health Initiative Observational Study: Baseline characteristics of participants and reliability of baseline measures. Ann Epidemiol 13 (Suppl): S107-S121, 2003.

29. Women's Health Initiative: About WHI: https://www.whi. org/about/SitePages/About\%20WHI.aspx. Accessed May 13, 2019.

30. Schachter M: Chemical, pharmacokinetic and pharmacodynamic properties of statins: An update. Fundam Clin Pharmacol 19: 117-125, 2005.

31. Desai P, Lehman A, Chlebowski RT, Kwan ML, Arun M, Manson JE, Lavasani S, Wasswertheil-Smoller S, Sarto GE, LeBoff M, et al: Statins and breast cancer stage and mortality in the Women's Health Initiative. Cancer Causes Control 26: $529-539,2015$.

32. Maringe C, Walters S, Rachet B, Butler J, Fields T, Finan P, Maxwell R, Nedrebø B, Påhlman L, Sjövall A, et al; ICBP Module 1 Working Group: Stage at diagnosis and colorectal cancer survival in six high-income countries: A population-based study of patients diagnosed during 2000-2007. Acta Oncol 52: 919-932, 2013.

33. Nayak J and Goel S: Revised TNM staging for colorectal cancer: Did we miss the golden opportunity to do right by the staging? Clin Colorectal Cancer 10: 207-209, 2011.

34. Katz MS, Minsky BD, Saltz LB, Riedel E, Chessin DB and Guillem JG: Association of statin use with a pathologic complete response to neoadjuvant chemoradiation for rectal cancer. Int J Radiat Oncol Biol Phys 62: 1363-1370, 2005.

35. Mace AG, Gantt GA, Skacel M, Pai R, Hammel JP and Kalady MF: Statin therapy is associated with improved pathologic response to neoadjuvant chemoradiation in rectal cancer. Dis Colon Rectum 56: 1217-1227, 2013.

36. Ng K, Ogino S, Meyerhardt JA, Chan JA, Chan AT, Niedzwiecki D, Hollis D, Saltz LB, Mayer RJ, Benson AB III, et al: Relationship between statin use and colon cancer recurrence and survival: Results from CALGB 89803. J Natl Cancer Inst 103: 1540-1551, 2011.

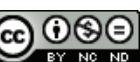

This work is licensed under a Creative Common Attribution-NonCommercial-NoDerivatives 4.0 International (CC BY-NC-ND 4.0) License. 